\title{
P01-8-41 Poster session
}

\section{Search for drugs that attenuate the antitumor effect of bevacizumab using adverse event database}

\section{Yoshito Zamami ${ }^{1,2}$, Yuki Izawa-Ishizawa ${ }^{3}$, Kenshi Takechi ${ }^{4}$, Masaki Imanishi ${ }^{2}$, Keijo Fukushima ${ }^{5}$,} Yuya Horinouchi ${ }^{3}$, Yasumasa Ikeda ${ }^{3}$, Hiromichi Fujino ${ }^{5}$, Toshiaki Tamaki ${ }^{3}$, Keisuke Ishizawa ${ }^{1,2}$

${ }^{I}$ Department of Clinical Pharmacology and Therapeutics, Institute of Biomedical Sciences, Tokushima University Graduate School, Japan, ${ }^{2}$ Department of Pharmacy, Tokushima University Hospital, Japan, ${ }^{3}$ Department of Pharmacology, Institute of Biomedical Sciences, Tokushima University Graduate School, Japan, ${ }^{4}$ Clinical Trial Center for Developmental Therapeutics, Tokushima University Hospital, Japan, ${ }^{5}$ Department of Molecular Pharmacology, Faculty of Pharmaceutical Sciences \& Institute of Biomedical Sciences, Graduate School, Tokushima University, Japan

BACKGROUND: Bacizumab is a monoclonal antibody against vascular endothelial cell growth factor (VEGF), one of antineoplastic agents that has indications for lung cancer, colorectal cancer and the like, but it is known to develop hypertension frequently as an adverse event. Meanwhile, it is reported that the onset of hypertension caused by bevacizumab is an indicator of antitumor effect. Therefore, a drug that suppresses bevacizumab-induced hypertension may attenuate the antitumor effect of bevacizumab. In the present study, we aimed to search for drugs that attenuate the antitumor effect of bevacizumab.

METHODS: Drugs that suppress bevacizumab-induced hypertension were extracted from the decrease in odds ratio of onset of hypertension due to the presence or absence of specific concomitant medications in bevacizumab administered cases using FDA Adverse Event Reporting System (FAERS) database. We also analyzed whether the drug found in the FAERS database attenuates the antitumor effect of bevacizumab in patients with colon \&\#183; rectal cancer administered with bevacizumab at the University of Tokushima hospital. Furthermore, whether the extracted drug acts on the VEGFrelated molecule or not is estimated by a pathway analysis using a gene expression database (GEO) and verified by an in vitro analysis using human colon cancer LS174T cells.

RESULTS: A comprehensive analysis of drugs that suppress bevacizumab-induced hypertension from among drugs used in combination with bevacizumab showed a tendency to suppress the onset of hypertension by combined administration simvastatin, loperamide, and metamizole, and a significant difference in omeprazole concomitant administration.

In patients with colorectal cancer who administered bevacizumab in combination with a proton pump inhibitor containing omeprazole, the rate of disease control was significantly lower than that in cases with no PPI combined. In addition, it was speculated that omeprazole may increase VEGF gene expression by pathway analysis, and the expression level of VEGF mRNA increased when colon cancer cells were stimulated with various PPIs.

CONCLUSIONS: It is suggested that various PPIs may attenuate the antitumor effect of bevacizumab through elevated VEGF expression. 